\title{
Estudo comparativo da fosfatização inorgânica e orgânica do aço carbono 1008 quanto à resistência a corrosão
}

\author{
Comparative study of inorganic and organic \\ phosphating of carbon steel 1008 regarding \\ resistance to corrosion
}

\author{
Marilei Fátima Oliveira ${ }^{1}$, Henrique de Santana ${ }^{2}$, \\ Marcelo Grassi ${ }^{3}$, Paulo Rogério Pinto Rodrigues ${ }^{3}$, \\ André Lanzarin Gallina ${ }^{3}$
}

\author{
${ }^{1}$ Departamento de Engenharia Mecânica - UTFPR - Rua Padre Salvador, n 875, Bairro Santa Cruz, 85015-430 \\ Guarapuava - Paraná, Brasil. \\ e-mail: ofmarilei@gmail.com \\ ${ }^{2}$ Departamento de Química da Universidade Estadual de Londrina, UEL- Rodovia Celso Garcia Cid, Pr 445 Km 380 \\ Campus Universitário da UEL, 86051-980 Londrina - Paraná, Brasil. \\ e-mail: hesan@uel.com.br \\ ${ }^{3}$ Departamento de Química da Universidade Estadual do Centro Oeste - Grupo de Pesquisa em eletroquímica, GPEL ${ }^{\circledR}$ \\ UNICENTRO - Rua Simeão Camargo Varela de Sá, n 03, Bairro Cascavel, 85040-080 - Guarapuava - Paraná, Brasil. \\ e-mail: marcelograssi22@gmail.com; prprodrigues@unicentro.br; andregallina@gmail.com
}

\section{RESUMO}

Devido às inúmeras aplicações do aço carbono na indústria, torna-se necessária a utilização de tratamento de superfície para controle e prevenção da corrosão deste metal. Um dos sistemas utilizados como prétratamento do aço carbono para posterior pintura é a fosfatização à base de zinco, a qual permite melhorar a ancoragem da tinta sobre o substrato metálico. No entanto, este tratamento gera para a indústria um passivo ambiental relacionado ao lodo e ao íon metálico alergênico e poluente utilizado no processo, neste caso o $\mathrm{Ni}^{2+}$. O objetivo deste trabalho é desenvolver e caracterizar um tratamento alternativo ao fosfato de zinco para o aço carbono 1008, utilizando-se um fosfato orgânico. O método utilizado foi o de imersão do substrato metálico em uma solução aquosa de uma molécula orgânica à temperatura ambiente. Os corpos de prova do aço carbono foram avaliados utilizando-se microscopia óptica e eletrônica de varredura, espectroscopia Raman, ensaios eletroquímicos de polarização potenciodinâmica anódica e espectroscopia de impedância eletroquímica em meios ácido e neutro, além dos ensaios em Salt Spray. Os resultados eletroquímicos e de corrosão acelerada mostraram que o filme de fosfato orgânico minimiza a corrosão no aço carbono, podendo ser utilizado em substituição ao tradicional fosfato de zinco.

Palavras- chave: Molécula auto organizável, fosfato de zinco, tratamento de superfície.

\section{ABSTRACT}

Due to the numerous applications of carbon steel in industry, the use of surface treatment to control and prevent the corrosion of this metal becomes necessary. One of the systems used as pre-treatment of carbon steel for subsequent painting is zinc phosphating, which allows to improve adhesion of ink onto the metal substrate. However, this treatment causes an environmental liability to industry, related to the silt and allergenic, pollutant metal ion used in the process, in this case $\mathrm{Ni}^{2+}$. The objective of this work is to develop and characterize a treatment alternative to zinc phosphate for the 1008 carbon steel, using an organic phosphate. The method used was that of immersing the metal substrate in an aqueous of an organic molecule at room temperature. Specimens of carbon steel were evaluated using optical microscopy and scanning electron microscopy, Raman spectroscopy, electrochemical tests of anodic polarization and electrochemical impedance spectroscopy in acidic and neutral medium, besides the Salt Spray tests. The electrochemical and fast corrosion 
results showed that the accelerated organic phosphate film minimizes the corrosion of the carbon steel, and it can be used instead of the traditional zinc phosphate.

Keywords: Molecule self-organizing, zinc phosphate surface treatment.

\section{INTRODUÇÃO}

O uso de metais na indústria em geral requer um estudo minucioso quanto às suas propriedades físicas e químicas, visando principalmente a resistência ao meio e as tensões mecânicas às quais é empregado [1, 2$]$.

$\mathrm{O}$ aço é uma liga ferrosa contendo até $2 \%$ de carbono. Devido à abundância do elemento ferro na crosta terrestre, esta liga torna-se uma das mais empregadas industrialmente, associada à economicidade e às suas propriedades químicas e físicas $[\underline{2}, \underline{3}]$.

A energia livre padrão da reação $\left(\Delta \mathrm{G}_{\mathrm{f}}^{\circ}\right) \mathrm{Fe}_{(\mathrm{s})} / \mathrm{Fe}_{2} \mathrm{O}_{3}$ é da ordem de $-742,2 \mathrm{kJmol}^{-1}$, demonstrando a espontaneidade natural das ligas ferrosas em se oxidarem [4]. A oxidação dessas ligas leva à deterioração do material por ação química ou eletroquímica do meio, aliada ou não a esforços mecânicos. Essa deterioração prejudica o desempenho do material, deixando-o muitas vezes impróprio para uso, pois leva à redução da resistência mecânica e à alteração da condutividade térmica, elétrica e do aspecto decorativo para algumas situações. Problemas de corrosão acontecem nas mais diversas atividades e, pelo fato de em muitas delas os investimentos serem de grande valor, a exigência é a de que o material seja resistente à corrosão e tenha uma grande durabilidade [5].

Para se minimizar a oxidação dessas ligas, torna-se necessário o uso de inibidores ou a utilização de processos que proporcionem a inibição da superfície, tais como: alteração do pH, alteração da temperatura, agitação, etc. [ $[\underline{5}, \underline{6}]$.

Os inibidores podem ser classificados como orgânicos e inorgânicos, além de seu caráter de adsorção química ou física [7].

A grande maioria dos inibidores inorgânicos (nitritos, cromatos, molibidatos, etc.) atua promovendo uma rápida oxidação do metal, dando origem a uma camada de óxidos que passa a ocupar o local dos sítios ativos de oxidação, reduzindo consideravelmente os processos anódicos []].

Os inibidores orgânicos apresentam algumas propriedades especiais em relação aos inibidores inorgânicos. Muitos inibidores orgânicos são substâncias com pelo menos um grupo funcional, responsável pela reação central do processo de adsorção física ou química. Neste caso, a força de ligação da adsorção química está relacionada ao tipo e densidade eletrônica do heteroátomo, bem como à polarizabilidade do grupo funcional $[\underline{9}, \underline{10}]$. Além dos inibidores e dos processos de inibição, há também os processos físicos de proteção da superfície metálica como, por exemplo, a aplicação de tintas, e os de conversão, como a fosfatização.

Normalmente, a inserção de uma camada inibidora entre o metal e o meio consiste na aplicação de um revestimento não metálico inorgânico, seguido pela aplicação de tinta [11]. Este método é considerado extremamente eficaz no combate ao processo destrutivo de corrosão. Um exemplo é a precipitação de compostos inorgânicos na superfície metálica de sais ligeiramente solúveis de fosfatos, em que é possível melhorar a resistência à corrosão.[12].

A fosfatização corresponde a um método de proteção de metais no qual a solução fosfatizante aplicada por aspersão, imersão ou por ambos os processos é normalmente constituída por água, ácido fosfórico livre, uma mistura de sais de fosfato de zinco, níquel, ferro ou manganês, e agentes oxidantes. Trata-se da conversão do metal em um fosfato (sal) insolúvel do íon metálico. O fosfato insolúvel deposita-se sobre o metal modificando as suas propriedades superficiais, conforme as reações de 1 a 4 [13] .

$$
\begin{aligned}
& \mathrm{H}_{3} \mathrm{PO}_{4(a q)}+2 \mathrm{H}_{2} \mathrm{O}_{(l)} \Leftrightarrow 2 \mathrm{H}_{3} \mathrm{O}_{(a q)}^{+}+\mathrm{HPO}_{4}{ }^{2-}{ }_{(a q)} \\
& \mathrm{Fe}_{(s)}+2 \mathrm{H}_{3} \mathrm{O}_{(a q)}^{+} \rightarrow \mathrm{Fe}^{2+}{ }_{(a q)}+2 \mathrm{H}_{2} \mathrm{O}_{(l)}+\mathrm{H}_{2(g)} \\
& \mathrm{Fe}^{2+}{ }_{(a q)}+\mathrm{HPO}_{4}{ }^{2-}{ }_{(a q)} \rightarrow \mathrm{FeHPO}_{4(s)} \\
& 3 \mathrm{Zn}^{2+}{ }_{(a q)}+2 \mathrm{PO}_{4}{ }_{(a q)}^{3-} \rightarrow \mathrm{Zn}_{3}\left(\mathrm{PO}_{4}\right)_{2(s)}
\end{aligned}
$$

A fosfatização é usada na indústria metalúrgica para tratar substratos como ferro, aço, aço galvanizado, alumínio e suas ligas [14]. A maior parte das carrocerias de automóveis é fosfatizada antes da pintura, para 
aumentar sua resistência à corrosão e a adesão da tinta. Outros tratamentos oferecem resistência à corrosão para metais não pintados [15].

Várias funções podem ser atribuídas à camada de fosfato "cristalina": a natureza não condutora dos cristais de fosfato confere ao substrato metálico uma propriedade de barreira com excelente proteção à corrosão [16]; os vazios entre os cristais podem fornecer ancoragem para camadas de pintura, aumentando a aderência da tinta; microporos entre os cristais da camada de fosfato expõem o metal, e isso é importante, para fornecer uma superfície condutora o suficiente para permitir a deposição catódica de primers, muito usados na indústria automotiva [17]. Para as tintas eletroforéticas, a literatura assegura que o processo de nucleação da tinta se inicia nestes microporos, onde há uma superfície metálica ativa, ou seja, sem a presença do fosfato, permitindo que a nucleação se expanda formando uma camada de tinta por toda a superfície do substrato contendo o filme de fosfato. [18].

Os sistemas de fosfatização utilizados atualmente atendem às necessidades da indústria em termos de proteção e resistência à corrosão, porém muitas questões são discutidas atualmente sobre estes processos, principalmente em termos do cumprimento de normas ambientais. Os principais problemas relacionados à utilização dos sistemas de fosfatização são [19]:

1. A presença de metais pesados nos efluentes do processo de fosfatização, como o níquel, que é um metal pesado e alergênico; e o cobre, que é utilizado como catalisador da reação;

2. A lama da fosfatização, que é constituída principalmente por fosfato férrico, mas pode conter outros fosfatos metálicos insolúveis que não conseguiram se fixar na superfície. A formação de lama na fosfatização é intrínseca e não pode ser evitada. Este resíduo acaba sendo mal destinado ou levado para aterros. Os fosfatos são poluentes agressivos das águas, pois podem causar o processo de eutrofização da água, causando desequilíbrio ambiental e morte de animais;

3. A necessidade de passivação da camada fosfatizada para uma boa resistência à corrosão.

Enfatiza-se a necessidade de se pensar em soluções para a redução da geração de resíduos na fonte e em alternativas capazes de possibilitar a reutilização de resíduos, substituição da matéria prima, criação de novos produtos e recuperação de energia, ou na adoção de tecnologias capazes de eliminar resíduos do processo [20].

Dentro deste contexto, têm sido oferecidas algumas propostas de tratamento para o aço carbono em substituição aos processos de fosfatização. Um dos primeiros processos desenvolvidos é baseado na utilização de fosfatos orgânicos, que podem ser obtidos pelo processo de plaforização, um tratamento de superfícies executado em um único estágio utilizando um polifosfato orgânico específico para formação de uma fina camada de resina de um polímero orgânico fosfatado. Esse processo utiliza solventes orgânicos para realizar a dissolução de óleos e gorduras presentes na superfície do aço carbono [21]. Essa dissolução acontece através da exposição da superfície do metal em câmara saturada por solventes orgânicos na forma de vapor.

Este é um processo difícil de ser utilizado pela indústria devido à dificuldade de aplicação e toxidez do mesmo, sendo que o metal após tratamento não consegue atender às especificações das indústrias em termos de resistência à corrosão.

O uso de moléculas que se auto-organizam, chamadas de SAM (self assembled monolayer), sobre uma superfície metálica está entre as alternativas tecnológicas que têm sido estudadas para a proteção contra corrosão de materiais metálicos [22].

Quando essas moléculas são aplicadas em superfícies metálicas, atuam como uma barreira, não permitindo que a umidade alcance o substrato metálico e, dessa forma, aumenta-se a resistência à corrosão do substrato metálico. Acredita-se que essas moléculas possam também atuar como promotoras de adesão entre revestimentos orgânicos (tinta) e o substrato metálico [23, 24].

Moléculas auto-organizáveis podem aderir-se à superfície via adsorção química, promovida por uma das extremidades polares de suas cadeias, formando sobre o substrato uma espécie de filme [25].

Do ponto de vista energético, a molécula auto-organizável empregada neste trabalho, Figura 1, pode ser dividida em três partes [26, 27]:

(A) A cabeça 1, a qual promove adsorção química ao substrato;

(B) O corpo da molécula, que é constituído de alcano, cujo número de carbonos varia dependendo do tipo de molécula, e

(C) a parte funcional da molécula, chamada de cabeça 2, que pode ser desde um grupo metil $\left(\mathrm{CH}_{3}\right)$ até outro composto específico, desenvolvido para dada aplicação. O espaçamento entre os grupos funcionais 
assegura o ordenamento e a estrutura compacta das camadas automontadas com interações de Van der Waals entre elas.

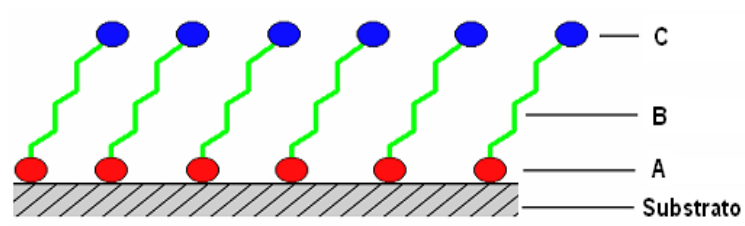

Figura 1: Esquema da adsorção de uma molécula auto-organizável (A-B-C) em um substrato, no qual (A) é a cabeça 1, (B) corpo e (C) cabeça 2 da molécula.

Para obtenção do filme de moléculas auto-organizáveis sobre a superfície do aço carbono, é necessário adequar corretamente as variáveis do processo de tratamento, a fim de possibilitar a auto-organização e uniformidade do filme sobre o substrato, pela formação de um fosfato de ferro. Diante do exposto, o objetivo deste trabalho foi avaliar a resistência à corrosão do filme formado pela molécula do ácido fosfonohexanóico (POr) sobre a superfície do aço carbono 1008, e comparar as propriedades deste filme com as propriedades de um filme de fosfato inorgânico à base de zinco (PZn), utilizado atualmente pela indústria.

\section{MATERIAIS E MÉTODOS}

\subsection{Materiais}

Foram utilizados como corpo de prova amostras de aço carbono 1008 de composição, conforme a Tabela $1[13]:$

Tabela 1: Composição química do aço carbono 1008

\begin{tabular}{l|l|l|l|l|l}
\hline ELEMENTO & $\mathbf{C}$ & Mn & P & S & Fe \\
\hline$\%(\mathrm{~m} / \mathrm{m})$ & 0,08 & 0,330 & 0,048 & 0,021 & 99,52 \\
\hline
\end{tabular}

As soluções empregadas para tratamento de amostras pelo sistema de fosfatização foram de grau comercial, sendo fornecidas pela empresa Tecnoquisa. No preparo destas soluções utilizou-se água destilada.

Os reagentes utilizados para preparo da solução de fosfato orgânico foram de grau analítico, sendo as soluções preparadas com a utilização de água ultrapura.

Após o tratamento das amostras, foi realizada a caracterização eletroquímica do revestimento obtido. Nesta caracterização, foi utilizada uma célula de três eletrodos, sendo eles:

Eletrodo de trabalho - amostras de aço carbono 1008 com e sem o tratamento com $0,68 \mathrm{~cm}^{2}$ de área;

Eletrodo de auxiliar - platina de grande área $\left(20 \mathrm{~cm}^{2}\right)$;

Eletrodo de referência - sulfato mercuroso (ESM) $\left(\mathrm{Hg} / \mathrm{Hg}_{2} \mathrm{SO}_{4}\right)$ para o meio $\mathrm{H}_{2} \mathrm{SO}_{4} 0,01 \mathrm{~mol} \mathrm{~L}{ }^{-1}$ e prata cloreto de prata $(\mathrm{Ag} / \mathrm{AgCl})$ para os ensaios em meio de $\mathrm{NaCl} 0,5 \mathrm{~mol} \mathrm{~L}^{-1}$.

\subsection{Tratamento do substrato}

Os corpos de prova do aço carbono 1008 foram previamente lixados com lixas de SiC \#320, \#400 e \#600, empregando-se uma politriz marca Arotec modelo APL-2, e posteriomente lavados com água e secos com ar frio.

O sequenciamento dos tratamentos de superfície dos corpos de prova do aço carbono 1008 é mostrado na Figura 2. 
OLIVEIRA, F. M., SANTANA, H., GRASSI, M., RODRIGUES,

P.R.P. GALLINA, A.L., revista Matéria, v.18, n.03, pp. 1395-1409, 2013.

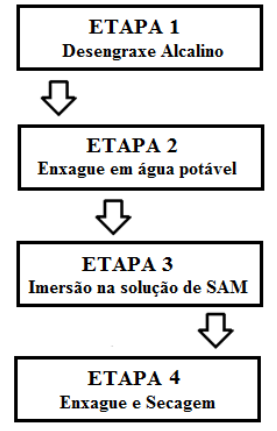

(A)

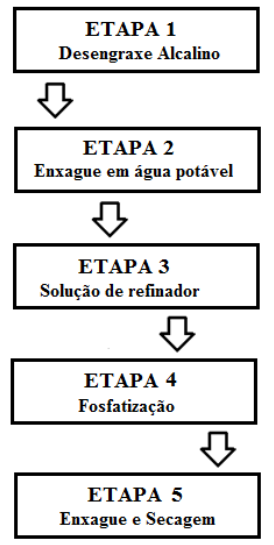

(B)

Figura 2: Tratamento das amostras de aço carbono. (A) Fosfato orgânico e (B) Fosfato de inorgânico (PZn).

No sistema proposto de formação do fosfato orgânico, assim como no sistema de fosfatização, o tratamento foi realizado pelo sistema de imersão do substrato nas soluções de tratamento.

Na primeira etapa, faz-se a imersão no desengraxante alcalino para remoção de oleosidade. O banho é preparado pela dissolução de $50 \mathrm{~g}$ do composto desengraxante em $1 \mathrm{~L}$ de água. As peças foram desengraxadas durante 5 minutos a uma temperatura de $80 \pm 5^{\circ} \mathrm{C}$. Estas condições de aplicação foram especificadas pelo fabricante do desengraxante.

Segunda etapa: enxague em água potável para simular as condições de aplicação industrial.

Terceira etapa: imersão na solução de SAM, para formação do fosfato orgânico POr. A molécula utilizada foi o ácido fosfonohexanóico, por um tempo de imersão de 5 a 10 minutos, sob temperatura ambiente $\left(20 \pm 5^{\circ} \mathrm{C}\right)$.

Quarta etapa: enxague em água potável e secagem a ar quente.

No tratamento de amostras de aço carbono pelo sistema de fosfatização inorgânica à base de sais de zinco e níquel, seguiu-se o fluxograma 2B nas condições especificadas pelo fabricante [28] .

Tabela 2: Delineamento fatorial para o efeito de temperatura, tempo de imersão e concentração da solução de POr sob o filme formado sobre aço carbono.

\begin{tabular}{l|l|l|l}
\hline NÚMERO DO EXPERIMENTO & $\mathbf{X}_{1}$ & $\mathbf{X}_{2}$ & $\mathbf{X}_{3}$ \\
\hline 1 & - & - & + \\
\hline 2 & + & + & + \\
\hline 4 & - & + & + \\
\hline 5 & + & - & + \\
\hline 6 & + & + & - \\
\hline 7 & - & + & - \\
\hline 8 & 0 & 0 & 0 \\
\hline 9 & + & - & - \\
\hline FATORES & - & - & - \\
\hline$X_{1}$ Concentração $\left(\mathrm{mol} \mathrm{L}{ }^{-1}\right)$ & 1 & - & \\
\hline$X_{2}$ Temperatura $\left({ }^{\circ} \mathrm{C}\right)$ & 70 & - & 0,001 \\
\hline$X_{3}$ Tempo de imersão $(\min )$ & 10 & 20 & 0,5 \\
\hline
\end{tabular}

Para determinar as melhores condições experimentais de temperatura, tempo de imersão e concentração para o tratamento das amostras com a solução de POr e para avaliar as condições que proporcionam o 
melhor filme, em termos de resistência à corrosão do metal base, foi realizada uma análise multivariada $2^{3}$ com ponto central, conforme a Tabela 2.

A análise multivariada é uma ferramenta estatística que permite a otimização experimental. Através do planejamento fatorial é possível avaliar simultaneamente o efeito de um grande número de variáveis, a partir de um número reduzido de experimentos. O planejamento estatístico do experimento permite avaliar não só a influência das variáveis no processo, mas suas interações, permitindo verificar também a confiabilidade dos resultados. A Tabela 2 mostra o delineamento fatorial obtido pelo uso do software Design Expert para um fatorial $2^{3}$.

\subsection{Caracterização Eletroquímica e Morfológica}

O comportamento eletroquímico das amostras foi avaliado por medidas de potencial de circuito aberto em função do tempo $\left(\mathrm{E}_{\mathrm{CA}}\right)$, curvas de polarização potenciodinâmica anódica e por espectroscopia de impedância eletroquímica (EIE), para os quais se utilizou um potenciostato da marca GAMRY modelo PCI-G300 e um analisador de frequência PCI - EIS300 acoplado a um computador.

As medidas de $\mathrm{E}_{\mathrm{CA}}$ foram realizadas até a estabilização do potencial, considerando-se a estabilidade como uma variação inferior a $5 \mathrm{mV}$ por pelo menos 30 minutos.

Os ensaios de polarização potenciodinâmica anódica foram realizados a partir do potencial de corrosão $\left(\mathrm{E}_{\text {corr }}\right)$ até sobretensão de $+200 \mathrm{mV}$, empregando-se uma velocidade de varredura de $1 \mathrm{mVs}^{-1}$. A polarização anódica cíclica foi realizada com velocidade de varredura de $1 \mathrm{mVs}^{-1}$, a partir do potencial de corrosão ( $\mathrm{E}_{\text {corr }}$ ) até o potencial de $1 \mathrm{~V}$ em relação ao eletrodo de $\mathrm{Ag} / \mathrm{AgCl}$, e retornando ao $\mathrm{E}_{\text {corr }}$.

As medidas de EIE foram realizadas potenciostaticamente no potencial de corrosão, com uma amplitude de perturbação de $\pm 10 \mathrm{mV}$, na faixa de frequência de $500 \mathrm{~Hz}$ a $25 \mathrm{mHz}$, utilizando-se 10 pontos por década para aquisição dos dados. Todos os ensaios eletroquímicos foram conduzidos à temperatura de $(20 \pm 5$ $\left.{ }^{\circ} \mathrm{C}\right)$.

As imagens de microscopia óptica das amostras antes e após a voltametria cíclica foram registradas através de um microscópio óptico marca Olimpus.

As análises de microscopia eletrônica de varredura (MEV) foram efetuadas em um microscópio da marca JEOL, modelo JSM7401F equipado com filamento de tungstênio de $25 \mathrm{kV}$ e um analisador de espectroscopia de energia dispersiva (EDS). Amostras diferentes foram analisadas, utilizando-se os aumentos de 1000 e $5000 X$.

\subsection{Análise Raman}

Os espectros Raman do fosfato orgânico foram obtidos de forma "ex situ" utilizando-se em Raman portátil Advantage532 $®$ da Delta $\mathrm{Nu}$, excitado em $\lambda=532 \mathrm{~nm}$ com resolução de $8 \mathrm{~cm}^{-1}$. Foi utilizado o software NuSpec da DeltaNu, usando recursos de linha de base para remover a fluorescência de fundo.

\subsection{Ensaios de Corrosão Acelerada}

Para os ensaios de névoa salina, utilizou-se o procedimento descrito pela norma ASTM B117 [29], empregando-se como solução cloreto de sódio a $5 \% \mathrm{~m} / \mathrm{m}$, em câmara de ensaio de salt spray marca Equilam série SS.

A área geométrica das amostras de aço carbono utilizada neste ensaio foi de $130 \mathrm{~cm}^{2}$. As peças foram pintadas com tinta eletrostática pó de base poliéster de cor branca. O revestimento orgânico foi riscado em forma de $\mathrm{X}$ com estilete até encontrar o substrato. As amostras foram expostas à névoa salina durante 500 horas. No teste de aderência, foi utilizada uma fita filamentosa, semitransparente, de $25 \mathrm{~mm}$ de largura, com adesividade de $(32 \pm 4) \mathrm{g} / \mathrm{mm}$ da $3 \mathrm{M}^{\circledR}$. A avaliação do resultado foi realizada considerando-se o desplacamento de tinta da área ensaiada, logo após a remoção da fita, utilizando o microscópio óptico. As amostras foram fotografadas para o registro e análise dos resultados.

\section{RESULTADOS}




\subsection{Planejamento Experimental}

Refere-se ao estudo da influência das variáveis, temperatura, tempo de imersão e concentração da solução de POr para tratamento do aço carbono.

A Tabela 3 apresenta os resultados do planejamento $2^{3}$ em termos da média da triplicata de cada ensaio em termos de resistência à polarização (Rp), obtidos nas medidas de EIE.

Tabela 3: Resultado de Rp em relação ao delineamento fatorial para o efeito de temperatura, tempo de imersão e concentração da solução de POr sob o filme formado sobre aço carbono.

\begin{tabular}{l|l}
\hline EXPERIMENTO & $\mathbf{R p}(\boldsymbol{\Omega} \mathbf{~ c m} 2)$ \\
\hline 1 & $308,7 \pm 55,3$ \\
\hline 2 & $189,8 \pm 33,1$ \\
\hline 3 & $184,0 \pm 43,6$ \\
\hline 4 & $\mathbf{4 0 4 , 1} \pm \mathbf{4 6 , 8}$ \\
\hline 5 & $206,0 \pm 56,5$ \\
\hline 6 & $273,4 \pm 60,3$ \\
\hline 7 & $220,9 \pm 28,0$ \\
\hline 8 & $127,9 \pm 18,5$ \\
\hline 9 & $138,8 \pm 55,5$ \\
\hline
\end{tabular}

A partir da Tabela 3 da análise da variância das respostas obtidas, os efeitos das variáveis $\mathrm{X}_{1}, \mathrm{X}_{2}$ e $\mathrm{X}_{3}$ apresentaram melhores resultados, em termos de resistência à polarização, no ensaio de número 4.

Para otimização do sistema já estudado para formação do fosfato orgânico na superfície do aço carbono, foi utilizado o ensaio 4 da Tabela 3 como ponto central, e realizado um novo planejamento experimental.

A Figura 3 ilustra a superfície de resposta da otimização do estudo referente ao planejamento fatorial do estudo das variáveis que influenciam no processo de formação do fosfato orgânico sobre a superfície de aço carbono.

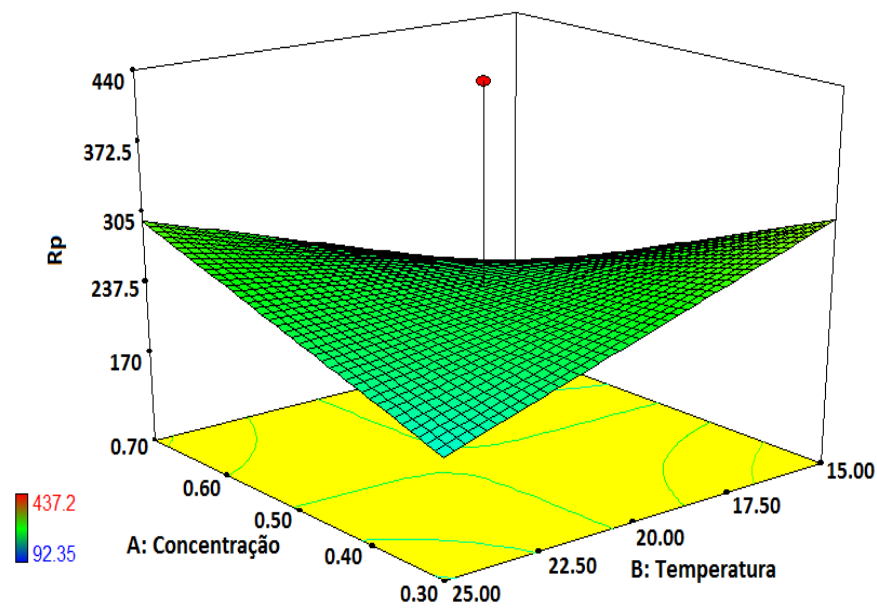

Figura 3: Superfície de resposta para a relação entre as variáveis, temperatura e concentração, para o tempo de imersão de $5 \mathrm{~min}$, em função da resistência à polarização $\left(\mathrm{Rp} / \Omega \cdot \mathrm{cm}^{2}\right)$.

De acordo com a Figura 3, observa-se que o ponto central (PC) tem um valor maior que os demais valores de superfície de resposta, sugerindo que este ponto (tempo de imersão de 5 minutos, temperatura de $20^{\circ} \mathrm{C}$ e concentração de $0,5 \mathrm{~mol} \mathrm{~L}^{-1}$ ) é o ponto em que se obtém o maior valor de Rp. Desta maneira, é comprovado que a melhor condição para formação do filme sobre o substrato de aço carbono é referente ao ensaio 4 da Tabela 3: 
Concentração da solução de POr $=0,5 \mathrm{~mol} \mathrm{~L}^{-1}$

Temperatura ambiente $=20 \pm 5 \mathrm{C}$

Tempo de imersão $=5$ minutos

\subsection{Caracterização em meio de $\mathrm{H}_{2} \mathrm{SO}_{4} 0,01 \mathrm{~mol} \mathrm{~L}^{-1}$}

Na Tabela 4 são mostrados os valores de potencial de corrosão para o aço carbono 1008 em meio de ácido $\mathrm{H}_{2} \mathrm{SO}_{4} 0,01 \mathrm{~mol} \mathrm{~L}^{-1}$.

Tabela 4: Valores de potencial de corrosão a $25^{\circ} \mathrm{C}$ dos corpos de prova do aço carbono 1008 em meio de $\mathrm{H}_{2} \mathrm{SO}_{4} 0,01$ mol $\mathrm{L}^{-1}$ com e sem o tratamento de fosfato orgânico.

\begin{tabular}{l|l|l|l}
\hline AMOSTRA & AÇO 1008 & AÇO 1008 + POr & AÇO 1008 + PZn \\
\hline $\mathrm{E}_{\text {corr }}(\mathrm{V}) / \mathrm{ESM}$ & $-0,947 \pm 0,026$ & $-0,976 \pm 0,01$ & $-0,978 \pm 0,03$ \\
\hline
\end{tabular}

Os resultados da Tabela 4 mostram que o $\mathrm{E}_{\text {corr }}$ com e sem o filme de fosfato orgânico tem pouca oscilação da ordem do $\mathrm{E}_{\text {corr }}=941 \pm 28 \mathrm{mV}$, demonstrando que, apesar da superfície metálica possuir um filme, o mesmo não provoca mudanças significativas em termos de potencial, e, portanto, no mecanismo de oxidação do substrato, isso se deve, principalmente, à alta porosidade do filme de POr obtido na superfície do substrato, conforme observado nas imagens de microscopia eletrônica de varredura.


(4B)

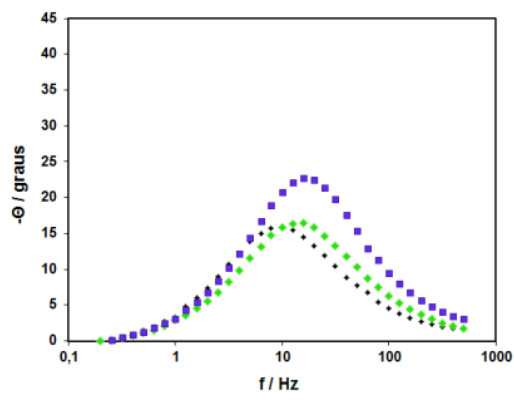

$(4 \mathrm{C})$

Figura 4: Em (A) Curvas de polarização potenciodinâmica anódica e (B) Diagramas de espectroscopia de impedância eletroquímica tipo Nyquist (C) tipo Bode. Em meio de $\mathrm{H}_{2} \mathrm{SO}_{4} 0,01 \mathrm{~mol} \mathrm{~L}^{-1}$ de corpos de prova de - aço carbono 1008 polido e tratado com: - POr e $-\mathrm{PZn}$

As curvas de polarização potenciodinâmica anódica e os diagramas de espectroscopia de impedância eletroquímica para o aço carbono 1008, apenas polido e tratado com POr e com PZ, são apresentados na figura 4A e 4B e apresentam o comportamento dos diferentes tratamentos em relação às reações de oxidação em $\mathrm{H}_{2} \mathrm{SO}_{4}$ 0,01 mol L $\mathrm{L}^{-1}$. Na polarização potenciodinâmica anódica, quando se aplica a sobretensão, tem-se a leitura da densidade de corrente (j), que mostra informações quanto à susceptibilidade à corrosão do material. Quanto maior a j obtida, maior é a velocidade de corrosão da amostra estudada. O comportamento observado na Figura 4A do corpo de prova de aço carbono 1008 contendo POr se deve ao bloqueio parcial da superfície do eletrodo provocado pela presença do filme, reduzindo a atividade oxidativa na amostra metálica e, portanto, promovendo menor densidade de corrente em todo o intervalo de potencial anódico estudado, quando comparado à amostra tratada com o PZn.

A análise do diagrama de impedância eletroquímica (Figura 4B) representa o estudo da interfase do 
eletrodo no meio em estudo, dada em termos de resistência à polarização, obtido pela perturbação no $\mathrm{E}_{\text {corr }}$. Quando foi realizado o tratamento do aço carbono com fosfato orgânico, a Rp aumentou consideravelmente em relação ao substrato apenas polido e tratado com fosfato de zinco. Isso significa uma maior resistência do substrato à oxidação quando na presença do filme de POr.

Os diagramas de ângulo de fase de Bode confirmam os resultados dos diagramas de Nyquist. A partir da Figura (4C) é possível observar apenas uma constante de tempo para todas as amostras. Para o aço carbono não revestido, a constante de tempo pode ser observada em frequências da ordem de $10 \mathrm{~Hz}$; para o aço carbono revestido com fosfato de zinco, há uma constante de tempo da ordem de $15 \mathrm{~Hz}$, a mesma ordem observada para o fosfato orgânico. A constante de tempo observada está relacionada aos processos de transferência de carga na superfície das amostras. Adicionalmente, um deslocamento para menores frequências foi observado para as amostras revestidas, o que sugere um retardamento do processo corrosivo.

\subsection{Caracterização em meio neutro}
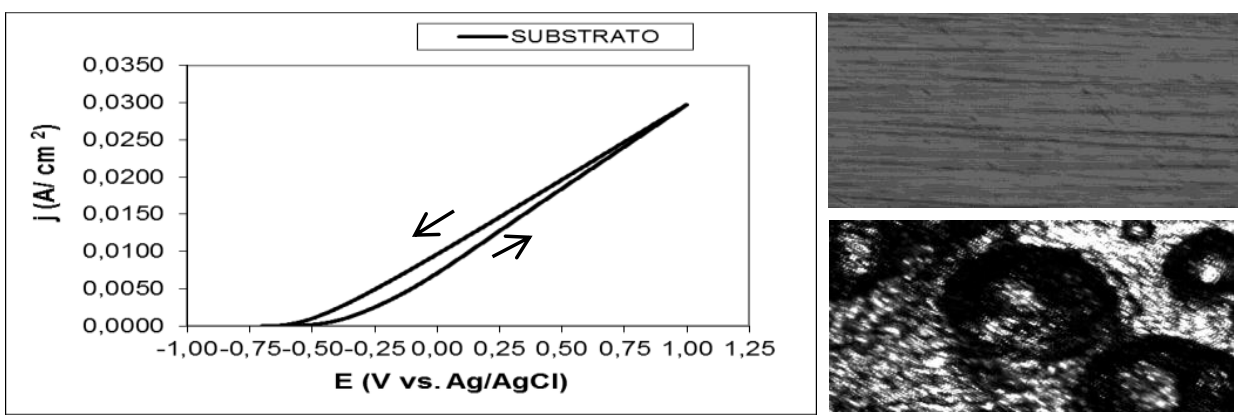

(A)
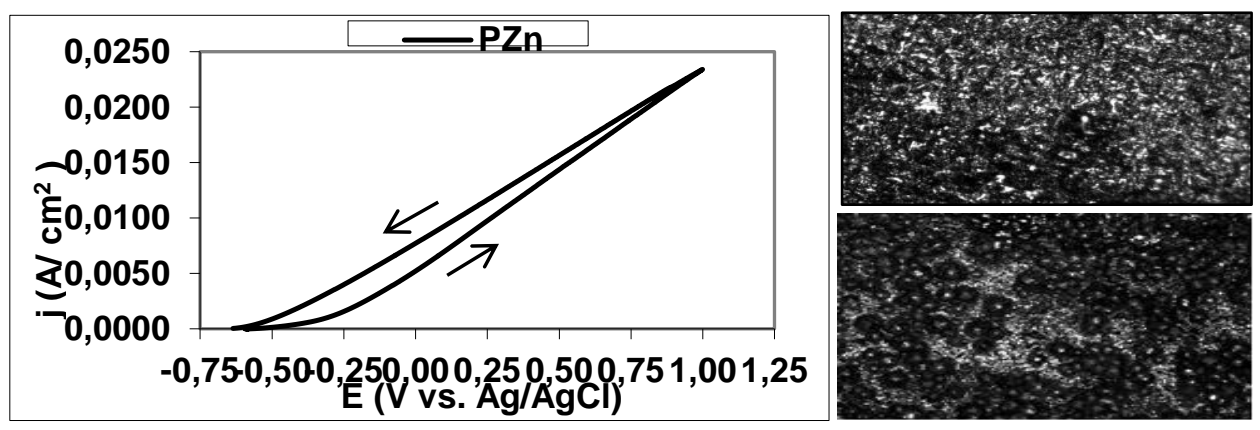

(B)

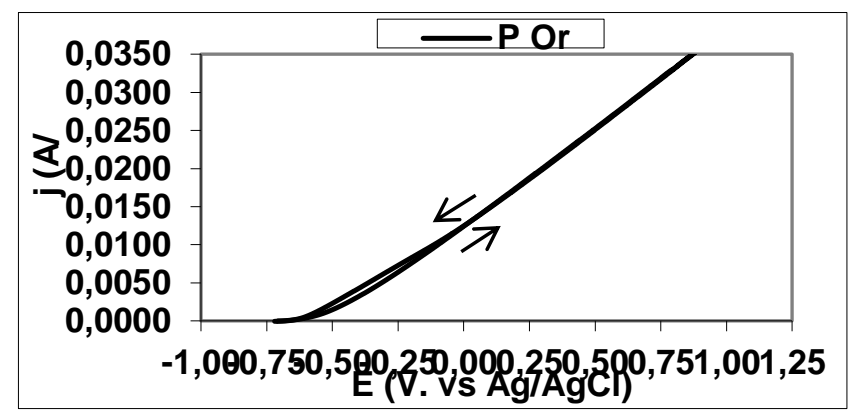

(C)
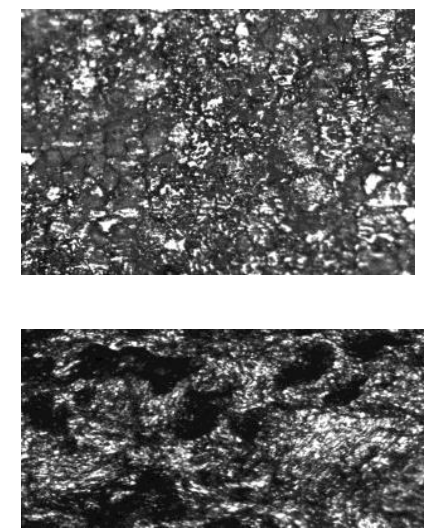

Figura 5: Curvas de polarização cíclica para o aço carbono 1008: (A) apenas polido, (B) tratado com PZn e (C) tratado com solução de POr em meio de $\mathrm{NaCl} 0,5 \mathrm{~mol} / \mathrm{L}$, v.v. de $1 \mathrm{mV} . \mathrm{s}^{-1}$. Microscopia óptica com aumento de $100 \mathrm{X}_{\text {no }} \mathrm{E}_{\text {corr }}$ após o ciclo anódico e retorno ao $\mathrm{E}_{\text {corr }}$ respectivamente.

As curvas de polarização anódica cíclica para amostras de aço carbono em meio de $\mathrm{NaCl} 0,5$ mol $\mathrm{L}^{-1}$ são 
apresentadas nas Figuras 5A, 5B e 5C. Microscopias ópticas da superfície dos corpos de prova foram feitas antes do ciclo de varredura anódica e após o retorno ao $\mathrm{E}_{\text {corr }}$, sendo apresentadas no inicio da curva anódica $(\rightarrow)$ e no retorno $(\leftarrow)$.

A análise de microscopia óptica da superfície do aço carbono 1008 na Figura 5A mostra que no retorno ao $\mathrm{E}_{\text {corr }}$ houve a formação de corrosão localizada na superfície metálica caracterizada por pontos alveolares, que sugerem corrosão por pite, causada por íons cloreto. Nota-se na Figura 5B, quando se faz o tratamento com zinco, que os pontos alveolares aumentam em quantidade, justificando a histerese na polarização cíclica, semelhante ao do substrato sem filme, ou seja, a camada de fosfato não consegue minimizar a migração de íons cloreto até a superfície do metal.

Ao se comparar a Figura 5C com a Figura 5A e B, nota-se que a histerese é muito menor em 5C, quando o substrato possui o filme de fosfato orgânico. Quando as curvas voltamétricas não apresentam tal histerese, é demonstrado que na superfície do material mesmo após a polarização não há mudança de área devido à corrosão localizada, ou seja, o filme é mais resistente a este tipo de corrosão.

Outra importante observação diferente da Figura 5 B, referente ao fosfato de zinco, é que a imagem morfológica mostrada na Figura 5C não apresenta características de ataques superficiais na forma de corrosão localizada; há apenas regiões mais escuras onde provavelmente houve a remoção do filme por processos oxidativos.

\subsection{Caracterização Morfológica}

São apresentadas na Figura 6 as imagem de microscopia eletrônica de varredura para as amostras de aço carbono apenas polido e amostra tratada com POr, respectivamente.

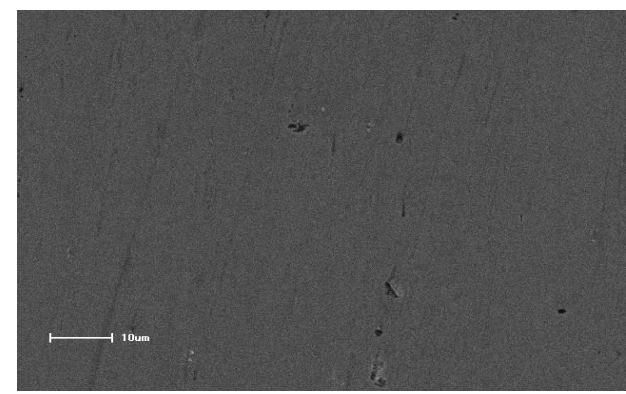

(6A)

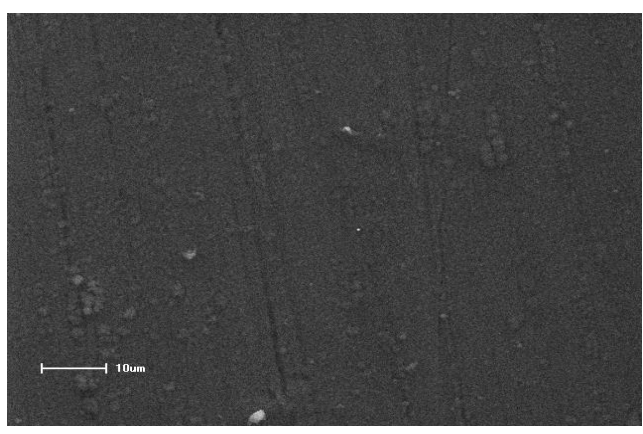

(6C)

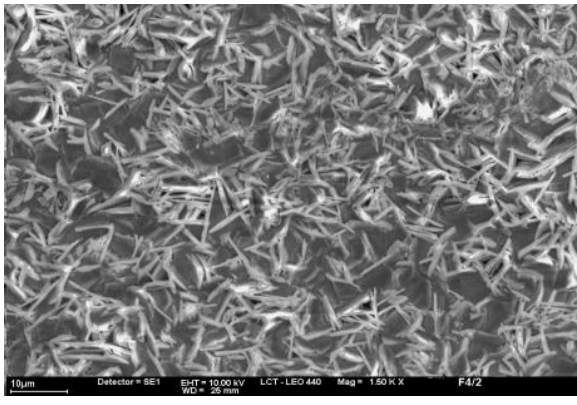

(6B)

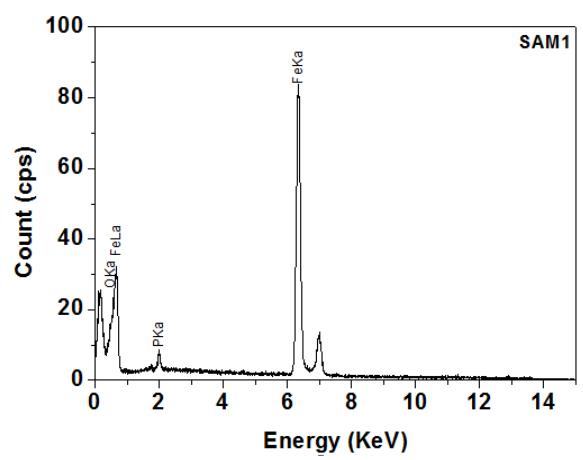

(6D)

Figura 6: Imagem de Microscopia eletrônica de varredura da superfície. Em (A), matriz de aço carbono 1008 apenas polido com aumento de 1000X; em (B), aço carbono tratado com PZn, aumento de 1500X; em (C), aço carbono tratado com POr aumento de 1000X; e, em (D), espectro de EDS da amostra de aço carbono tratado com POr.

Observa-se na Figura 6A que a superfície do aço carbono 1008 apresenta pequenos "buracos", que podem ser atribuídos às inclusões arrancadas no polimento da amostra. Quando se compara a amostra da Figura 6A com a amostra da Figura 6C, é possível observar o depósito de fosfato orgânico na superfície em forma de aglomerados. Pode-se comprovar a presença da molécula auto-organizável por EDS, referente ao elemento fósforo da Figura $6 \mathrm{C}$, constituinte da molécula $\mathrm{C}_{6} \mathrm{H}_{13} \mathrm{O}_{5} \mathrm{P}$. 
OLIVEIRA, F. M., SANTANA, H., GRASSI, M., RODRIGUES,

P.R.P. GALLINA, A.L., revista Matéria, v.18, n.03, pp. 1395-1409, 2013.

Também é importante ressaltar a característica do filme cristalino de fosfato de zinco na forma de agulhas de 7 a $10 \mu \mathrm{m}$ de largura [28].

\subsection{Espectroscopia Raman} com POr.

Na Figura 7, tem-se o espectro de Raman de amostra da POr pura e do aço carbono, quando tratado

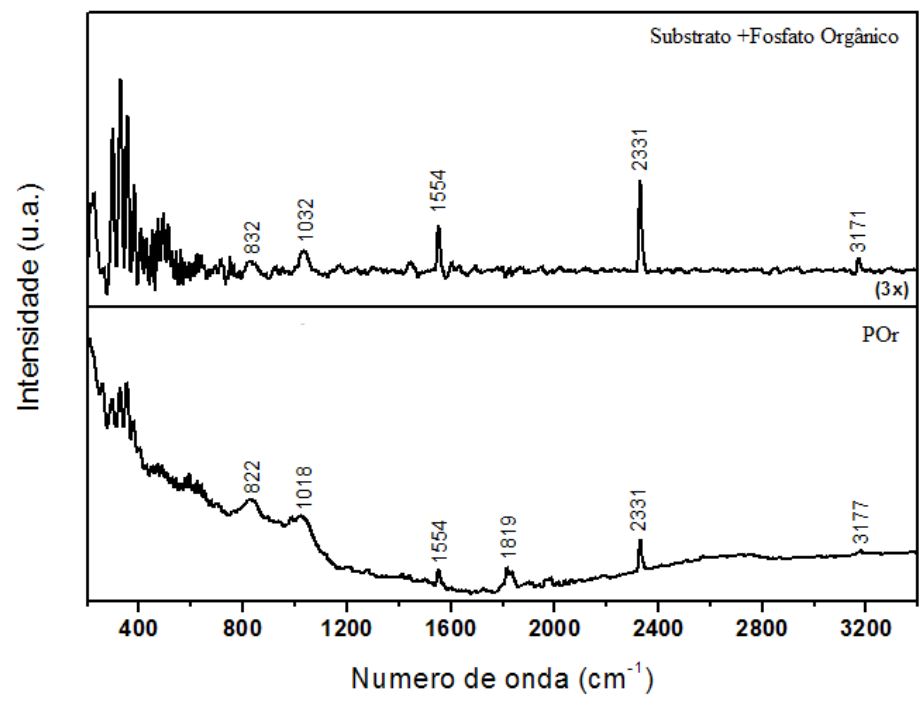

Figura 7: Espectros Raman de amostra da molécula de POr pura (pó) e do aço carbono tratado com solução de POr.

Ao avaliar os espectros da Figura 7, é possível sugerir que realmente tem-se a molécula autoorganizável sobre a superfície metálica, pois os picos de absorção das ligações da molécula POr pura correspondentes no espectro da amostra do aço quando tratado com POr.

$\mathrm{O}$ estiramento axial da hidroxila ligada ao átomo de fósforo $\mathrm{OH}-(\mathrm{POH})$ encontra-se em $2331 \mathrm{~cm}^{-1}$, presente no espectro da amostra de PO pura e também na amostra do aço carbono tratado como POr. Segundo a literatura [30], em espectroscopia de infravermelho de amostras de cristais de fosfato de zinco $\left(\mathrm{PZn} . \mathrm{H}_{2} \mathrm{O}\right)$ sobre PET, obtém-se pico intenso nesta região.

Em relação ao grupo fosfato, dois principais modos vibracionais são o estiramento simétrico próximo a $1095 \mathrm{~cm}^{-1}$ e a vibração assimétrica O-P-O localizada em $832 \mathrm{~cm}^{-1}$.

Ao ampliar o espectro da Figura 7 nas regiões entre 1600 e $1400 \mathrm{~cm}^{-1}$, é possível mostrar o estiramento da ligação C-C, presente entre os 5 carbonos que compõem a molécula auto-organizável em $1554 \mathrm{~cm}^{-1}$ (Figura 8A).

A banda situada em $3177 \mathrm{~cm}^{-1}$ correspondente ao estiramento axial $\mathrm{OH}$, é melhor visualizada quando se amplia esta região do espectro, na Figura 8B.

A Tabela 5 apresenta as principais atribuições dos espectros de Raman da amostra de aço carbono 1008 tratada com POr.

Tabela 5: Principais bandas observadas nos espectros de Raman e atribuições, dos espectros da Figura 7.

\begin{tabular}{l|l}
\hline$\lambda\left(\mathbf{c m}^{-1}\right)$ & DIMENSÃO \\
\hline $3177-3171$ & v O-H \\
\hline 2331 & OH-(POH) \\
\hline 1554 & C-C \\
\hline $832-822$ & v O-P-O \\
\hline $300-600$ & Região de óxidos \\
\hline
\end{tabular}

$v$-estiramento axial; $\delta$ - deformação angular 
OLIVEIRA, F. M., SANTANA, H., GRASSI, M., RODRIGUES,

P.R.P. GALLINA, A.L., revista Matéria, v.18, n.03, pp. 1395-1409, 2013.

Após a comprovação da deposição da molécula auto-organizável, é proposto o mecanismo representado nas equações 5 a 8 para formação do fosfato orgânico na superfície do aço carbono 1008 .
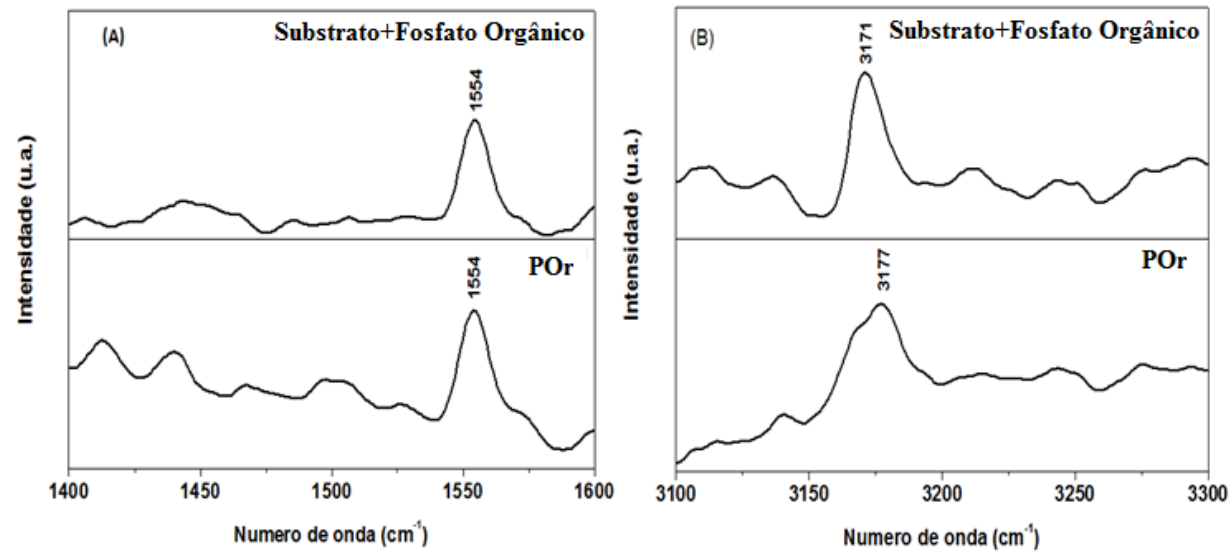

Figura 8: Ampliação dos espectros Raman de amostra da POr (pó) e do aço carbono contendo o filme POr.

No contato do substrato metálico com a solução da molécula auto-organizável, primeiramente ocorre o ataque da superfície metálica e a formação de íons ferro (II), que deve ocorrer de forma rápida (reação 5), e, na sequência, há a desprotonação do ácido fosfonohexanóico e complexação dos íons ferro (III). ou seja, as reações 6 a 8 ocorrem em uma única etapa. A nucleação da molécula auto-organizável foi acompanhada pela medida de estabilização de potencial de circuito aberto, em que se registrou a estabilização em até 5 minutos; ou seja, para que a molécula tenha se auto-organizado na superfície metálica em tão pouco tempo, é necessário que a oxidação da superfície aconteça de forma rápida, para então ocorrer sua nucleação. Dessa forma, pode-se considerar que as reações 6 a 8 ocorreram rapidamente, vide Figura 9.

Esta proposta de mecanismo (reações 5 a 8) para formação do fosfato orgânico de ferro sobre a superfície do aço carbono justifica a insignificativa variação do $\mathrm{E}_{\text {corr }}$ e os diagramas de impedância eletroquímica de Bode, que apresentam uma constante de tempo semelhante ao sistema de fosfatização de zinco, o qual apresenta quatro reações (reações 1 a 4).

$$
\begin{aligned}
& \mathrm{Fe}_{(s)} \rightarrow \mathrm{Fe}^{2+}{ }_{(a q)}+2 e^{-} \\
& \mathrm{C}_{6} \mathrm{H}_{13} \mathrm{PO}_{5(s)} \rightarrow \mathrm{C}_{6} \mathrm{H}_{12} \mathrm{PO}_{5}^{-{ }_{(a q)}+\mathrm{H}^{+}{ }_{(a q)}} \\
& \mathrm{C}_{6} \mathrm{H}_{13} \mathrm{PO}_{5(s)} \rightarrow \mathrm{C}_{6} \mathrm{H}_{12} \mathrm{PO}_{5}^{-{ }_{(a q)}}+\mathrm{H}^{+}{ }_{(a q)} \\
& 3\left(\mathrm{C}_{6} \mathrm{H}_{12} \mathrm{PO}_{5}^{-}\right)_{(a q)}+\mathrm{Fe}^{3+}{ }_{(a q)} \rightarrow \mathrm{Fe}\left[\mathrm{C}_{6} \mathrm{H}_{12} \mathrm{PO}_{5}\right]_{3(s)}
\end{aligned}
$$

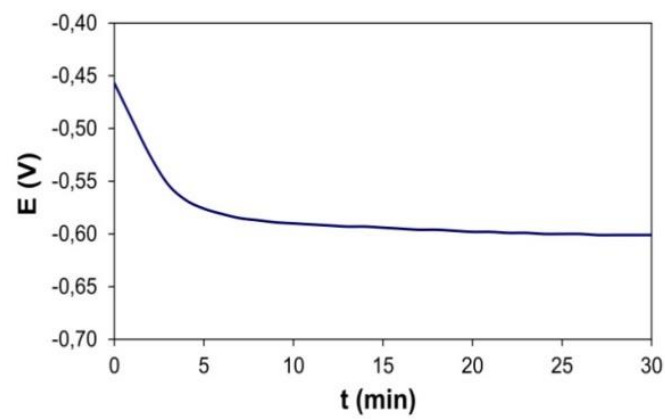

Figura 9: Medidas de potencial de corrosão vs. eletrodo de referência $(\mathrm{Ag} / \mathrm{AgCl})$, a $25^{\circ} \mathrm{C}$ de amostra de aço carbono imerso na solução da molécula orgânica sob temperatura ambiente $\left(22,5 \pm 5^{\circ} \mathrm{C}\right)$. 


\subsection{Ensaios de corrosão acelerada- névoa salina}

Ensaios de névoa salina foram realizados para avaliação da resistência à corrosão de amostras que, após o tratamento, foram pintadas com tinta pó poliéster de cor branca.

As Figuras 10A e B mostram os resultados da análise de salt spray em câmara salina com solução neutra de $\mathrm{NaCl} 5 \%$ para as amostras de aço carbono tratado pelo processo de fosfatização à base de zinco e pelo processo proposto POr.

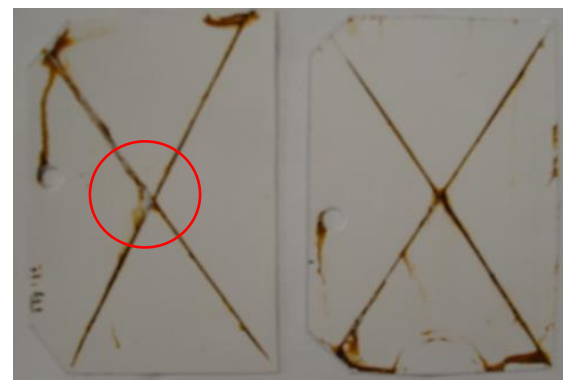

$(10 \mathrm{~A})$

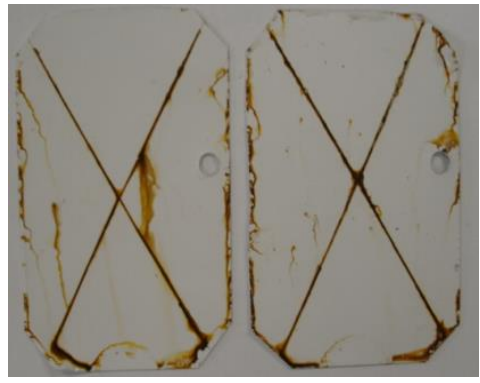

$(10 \mathrm{~B})$

Figura 10: Fotografias do resultado da análise de salt spray pelo período de 500 h, para amostra de aço carbono 1008. (A) com PZn e (B) com POr. Os corpos de prova foram pintados com tinta eletrostática pó poliéster branca.

Quando se compara a oxidação na região do corte das amostras tratadas com PZn e POr nas Figuras 10A e B, observa-se que, na amostra tratada com o POr, não ocorre desplacamento da tinta em nenhuma região do corte e, portanto, não houve corrosão filiforme, demonstrando que o filme de fosfato orgânico formado permite boa ancoragem da tinta sobre o substrato metálico.

A amostra tratada com PZn apresentou desplacamento em algumas regiões do corte, resultado da oxidação do substrato. Quando a névoa salina penetra no revestimento e atinge a interface substratorevestimento, provoca a perda de adesão da tinta e, assim, o seu desplacamento. A perfeita adesão entre a tinta e o substrato depende das propriedades da tinta pó, mas é o revestimento de conversão que tem o papel de melhorar a aderência da camada de tinta sobre a superfície do metal. Neste caso, em que se tem um filme cristalino de fosfato de zinco na superfície em forma de agulhas, vide Figura 6 B, é possibilitada a infiltração da névoa salina e o contato direto de íons cloreto com a superfície do aço carbono, levando ao desplacamento da tinta.

\section{CONCLUSÕES}

$\mathrm{O}$ filme de fosfato orgânico se mostrou mais eficiente na proteção à oxidação do aço carbono em $\mathrm{H}_{2} \mathrm{SO}_{4} 0,01$ mol L $\mathrm{L}^{-1}$, apresentando no diagrama de EIE um arco de impedância com o dobro da resistência registrada em relação ao substrato sem fosfatização.

Os ensaios eletroquímicos em $\mathrm{NaCl} 0,5 \mathrm{~mol} \mathrm{~L}^{-1}$ mostraram que o substrato de aço carbono 1008 com filme de fosfato orgânico não apresenta histerese significativa na polarização cíclica anódica, ou seja, não houve mudança expressiva da área do eletrodo que pudesse justificar a existência de corrosão localizada.

Os ensaios de corrosão acelerada mostraram que o tratamento de superfície com POr permite a ancoragem da tinta pó, oferecendo melhores propriedades à resistência à corrosão do que o tratamento com fosfato de zinco.

As medidas de $E_{\text {corr }}$ não mostram alterações significativas em seus valores, e as de EIE mostram que os mecanismos de geração do fosfato de zinco ou do fosfato orgânico sobre a superfície do aço carbono, devido à similaridade nos diagramas tipo Bode, ocorrem em mesma quantidade de constantes de tempo, sugerindo similaridade no processo reacional de formação dos filmes (reações 5 a 8).

A utilização de um tratamento orgânico para preparação do aço carbono para recebimento de tinta se mostrou positiva em termos de resistência à corrosão do aço carbono e à ancoragem da tinta epóxi, além de ser menos agressiva ao meio ambiente do que o fosfato de zinco, por não produzir lodo similar ao do fosfato 
OLIVEIRA, F. M., SANTANA, H., GRASSI, M., RODRIGUES,

P.R.P. GALLINA, A.L., revista Matéria, v.18, n.03, pp. 1395-1409, 2013.

de zinco e não utilizar no processo orgânico íons alergênicos como, por exemplo, os íons $\mathrm{Ni}^{2+}$.

\section{AGRADECIMENTOS}

Ao CNPq, Capes, FINEP e à Fundação Araucária, pelo suporte financeiro a esta pesquisa.

\section{BIBLIOGRAFIA}

[1] PANNONI, D.F., "Fundamentos da Corrosão”, Pintura Industrial, v. 48, n.16, pp. 32-35, 2007.

[2] GENTIL, V., Corrosão, 3 ed., Rio de Janeiro, Guanabara Dois S.A., 1982.

[3] CHIAVERINI, V., Aços e Ferros Fundidos: características gerais, tratamentos térmicos, principais tipos, 6 ed., São Paulo, ABM, 1988.

[4] LIDE, D.R., Handbook of Chemistry and Physics, 82 ed., Boca Raton, Flórida, USA, CRC Press, $2001-2002$.

[5] CUNHA, M.T , RODRIGUES, P.R.P., CORDEIRO, G.G.O., et al., "Electrochemical studies of the interface Fe/0.5 $\mathrm{mol} \mathrm{L}^{-1} \mathrm{H}_{2} \mathrm{SO}_{4}$ in the presence of benzotriazole and tolytriazole", Materials Chemistry and Physics, v. 116, n.2-2, pp. 469-473, 2009.

[6] MENNUCCI, M.M., BANCZEK, E.P., RODRIGUES, P.R.P., et al.,"Evaluation of benzotriazole as corrosion inhibitor for carbon steel in simulated pore solution", Cement \& Concrete Composites, v. 31, n.6, pp. 418-424, 2009.

[7] TUSSOLINI, M., SPAGNOL, C., GOMES, E.C., et al., "Estudo do comportamento eletroquímico do benzotriazol e benzimidazol na oxidação do aço inoxidável tipo ABNT 304”, REM - Revista da Escola de Minas, v. 60, n. 1, pp. 41-44, 2007.

[8] RODRIGUES, P.R.P., ZERBINO, J.O., AGOSTINHO, S.M.L, "Voltammetric and ellipsometric studies of films formed on 304 stainless steel in sulphuric acid solution without and with benzotriazole", Materials Science Forum, v. 288, pp. 1299-1310, 1998.

[9] CARDOSO, S.P., REIS, F.A., MASSAPUST, F.C., et al., "Avaliação de indicadores de uso diverso como inibidores de corrosão", Química Nova, v. 28, n. 5, pp. 756-760, 2005.

[10] BANCZEK, E.P, OLIVEIRA, M.F., CUNHA, M.T., et al., "Study of the Electrochemical Behaviour of Tolitriazole in Phosphating Bathings of Carbon Steel 1008”, Portugaliae Electrochimica Acta, v. 23, n. 3, pp. 379-391, 2005.

[11] BANCZEK, E.P., RODRIGUES, P.R.P., COSTA, I., "Evaluation of porosity and discontinuities in zinc phosphate coating by means of voltametric anodic dissolution (VAD)", Surface and Coatings Technology, v. 203, n.9, pp. 1213$1219,2009$.

[12] DONOFRIO, J., “Zinc phosphating”, Metal Finishing, v. 98, n. 6, pp. 57-8, 60-73, 2000.

[13] BANCZEK, E.P., Desenvolvimento e Caracterização de Camadas de Fosfato de Zinco/Níquel e Zinco/Nióbio, Tese de D. Sc., IPEN/CNENSP, São Paulo, SP, Brasil, 2008.

[14] OLIVEIRA, F.M., Tratamento de superfície fosfatização, Monografia Especialização em Química, UNICENTRO, Guarapuava, PR, Brasil, 2003.

[15] NARAYANAN, T.S.N.S., "Performance evaluation of phosphating formulations in continuous operation", Metal Finishing, v. 94, n. 9, pp. 40,42-43, 1996.

[16] BANCZEK, E.P, RODRIGUES, P.R.P., COSTA, I., "Determinação quantitativa da porosidade de camadas de fosfato de zinco", Tratamento de Superfície, v. 160, p. 42-49, 2010.

[17] TESTA, A., “Camadas de conversão nanocerâmicas”, Tratamento de Superfície, n. 130, p. 38-43, 2005.

[18] VIEIRA, A.C.S., ARAMAKI, S.M., "Técnicas de proteção superficial em metais: pintura eletroforética catódica e pintura a pó”, Tratamento de Superfície, n. 136, p. 30-35, 2006.

[19]WENG, D, WANG, R. ZHANG, G., "Environmental impact of zinc phosphating in surface treatment of metals", Metal Finishing, v. 96, n. 9, p. 54, 56-57, 1998.

[20] PANOSSIAN, Z., SANTOS, C.A.L., "Fosfatização de Metais Ferrosos - Parte 2 - Tipos de Camadas Fosfatizadas", Corrosão \& Proteção, v. 3, n.11, pp. 24-26, 2006.

[21] USINA DE LETRAS, http://www.usinadeletras.com.br/exibelotexto.php?cod=17189\&cat=Artigos\&vinda=S, acessado em novembro de 2012 .

[22] MORAIS, R.F., Investigação do tratamento com moléculas auto organizáveis para substituição da fosfatização da liga AA5052H32 no preparo da superfície prévio a pintura, Tese de M.Sc., IPEN/CNENSP, São Paulo, SP, Brasil, 2005. 
OLIVEIRA, F. M., SANTANA, H., GRASSI, M., RODRIGUES,

P.R.P. GALLINA, A.L., revista Matéria, v.18, n.03, pp. 1395-1409, 2013.

[23] ARAMAKI, K., SHIMURA, T., "Preparation of a one-dimensional polymer film on passivated iron by modification of a carboxylate ion self-assembled monolayer with octyltriethoxysilane for preventing passive film breakdown", Corrosion Science, v. 46, n. 10, pp. 2533-2548, 2004.

[24] MAEGE, I , JAEHNE, E., HENKE, A., et al., "Self-assembling adhesion promoters for corrosion resistant metal polymer self-assembling adhesion promoters for corrosion resistant metal polymer interfaces", Progress in Organic Coatings, v. 20, n.1-4, pp.1-12, 1997.

[25] ULMAN, A., "Formation and structure of self assembled monolayers", Chemical Reviews, v. 96, n. 4, pp.1533-1554, 1996.

[26] BOSSARDI, K., Nanotecnologia aplicada a tratamento superficiais para o aço carbono 1020 como alternativa ao fosfato de zinco. Dissertação M.Sc., PPGEM/UFRGS, Porto Alegre, RS, Brasil, 2007.

[27] BAIN, C.D., "Formation of monolayer films by spontaneous assembly of organic thiols from solution onto gold", Journal of the Chemical Society, v. 111, n. 1, pp. 321-335, 1989.

[28] OLIVEIRA, F.M., Processo de fosfatização a base de ferro II contendo tolitriazol para o aço carbono, Tese de M.Sc., UNICENTRO, Guarapuava, PR, Brasil, 2008.

[29] AMERICAN SOCIETY FOR TESTING AND MATERIALS Standard Test Method of Salt Spray (Fog) Testing, Philadelphia, ASTM, 1990.

[30] BRANDÃO, S.L. Síntese e caracterização de nanocompósitos de pet com fosfatos híbridos de zircônio lamelares. Tese de D. Sc. UFRJ/IMA, Rio de Janeiro, Brasil, 2006. 